C-A/AP/\#174 October 2004

\title{
Possible phase loop for the global betatron decoupling
}

\author{
Y. Luo, P. Cameron, S. Peggs, D. Trbojevic
}

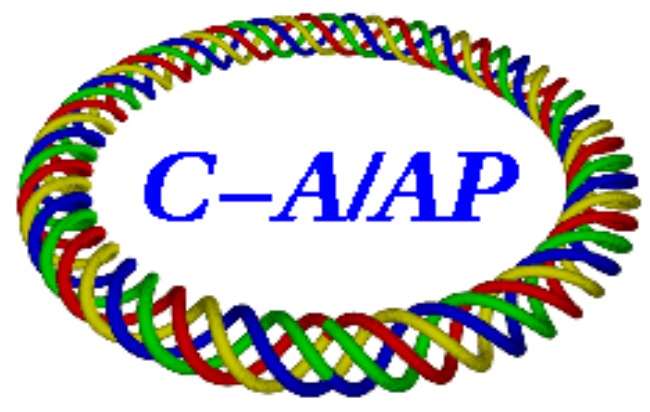

Collider-Accelerator Department Brookhaven National Laboratory Upton, NY 11973 
$\mathrm{CAD} / \mathrm{AP} / 174$

October 4, 2004

\title{
Possible phase loop for the global betatron decoupling
}

\author{
Y. Luo, P. Cameron, S. Peggs, D. Trbojevic \\ Brookhaven National Laboratory, Upton, NY 11973, USA
}

This paper suggests a possible phase loop for the global betatron decoupling.

The high resolution phase lock loop (PLL) system supplies 6 well-defined quantities: two eigentunes $Q_{1}$ and $Q_{2}$, and two amplitude ratios $r_{1}$ and $r_{2}$, two phase differences $\Delta \phi_{1}$ and $\Delta \phi_{2}$. The two eigenmode's tune split $\left|Q_{1}-Q_{2}\right|$ has been conventionally used for the global betatron coupling measurements and corrections. Here the other four quantities are looked into based on the free oscillation.

In the simulation based on the smooth accelerator model, these 6 quantities' changes are observed in the process of the skew quadrupole strength scans. Some attractive features of the phase differences $\Delta \phi_{1,2}$ have been found, which are possibly useful for the global decoupling phase loop, or global decoupling feedback.

These 4 quantities can be described in the Twiss and coupling parameters using linear coupling's actionangle parameterization. However, we find that linear coupling's Hamiltonian perturbation theory by G. Guignard is more straightforward to connect these quantities to the individual skew quadrupoles. Analytical descriptions of the 6 quantities have been obtained in that theory.

All the simulation and conclusions given in this note are based on the free oscillation. How to extend them to the PLL driving oscillation is still under investigation. The result will be given in the following AP notes.

\section{Observation of global decoupling scan}

In the section we will define 6 observable quantities of the global coupling. Simulations are carried out to observe their changes during the skew quadrupole scans. Some interesting and attractive features of the phase differences have been found, which are possibly useful for the future global decoupling phase loop.

\subsection{6 quantities}

For the linearly coupled circular accelerators, it is known that the displacements $x$ and $y$ of the free oscillating particle include the contributions from both eigenmodes. Here we assume that mode I is more linked to the horizontal plane, and mode II is more linked to the vertical plane. Then the particle's $n$th turn $x$ and $y$ coordinate can be casted as

$$
\left\{\begin{array}{l}
x_{n}=A_{1, x} \cos \left[2 \pi Q_{1}(n-1)+\phi_{1, x}\right]+A_{2, x} \cos \left[2 \pi Q_{2}(n-1)+\phi_{2, x}\right] \\
y_{n}=A_{1, y} \cos \left[2 \pi Q_{1}(n-1)+\phi_{1, y}\right]+A_{2, y} \cos \left[2 \pi Q_{2}(n-1)+\phi_{2, y}\right]
\end{array},\right.
$$

where $Q_{1}$ and $Q_{2}$ is the two eigentunes for the two eignmodes. The amplitude and initial phases both have subscripts $(i, j)$. The first subscript represents mode I or mode II, the second represents their contributions in the horizontal or vertical plane.

Besides the two eigentunes $Q_{1}$ and $Q_{2}$, here we define another 4 observable quantities to describe the global coupling. $r_{1}$ and $r_{2}$ are the amplitude ratios between the contributions from mode I or mode II in the two planes,

$$
\left\{\begin{array}{l}
r_{1}=\left|A_{1, y}\right| /\left|A_{1, x}\right| \\
r_{2}=\left|A_{2, x}\right| /\left|A_{2, y}\right|
\end{array} .\right.
$$

Under the uncoupled situation, $r_{1,2}=0$.

$\Delta \phi_{1}$ and $\Delta \phi_{2}$ are the phase differences between the contributions from mode I or mode II into the horizontal and vertical planes,

$$
\left\{\begin{array}{l}
\Delta \phi_{1}=\phi_{1, y}-\phi_{1, x} \\
\Delta \phi_{2}=\phi_{2, x}-\phi_{2, y}
\end{array} .\right.
$$


Under the uncoupled situation, $\Delta \phi_{1}$ and $\Delta \phi_{2}$ have not meanings since no mode I projection in y plane and no mode II projection in x plane.

With high performance digital BPMs and the modern analytical techniques $[1,2,3,4,5]$, the betatron phases $\phi_{i, j}$ can be very precisely measured in the beam experiments. The merit of the phase measurement is that it is independent from BPM position offsets and their gains. For a specific betatron oscillation, the two eigenmodes' phases therefore their differences $\Delta \phi_{1}$ and $\Delta \phi_{2}$ are all well defined. It will be very attractive if we can link the phase differences to the global coupling.

\subsection{Simulation and data processing}

Here we first give some simulation results based on the smooth accelerator model [6]. The model is simple but useful for the theoretical study and it gives very quick result comparing to the element-by-element tracking.

Using the smooth accelerator model, one particle's uncoupled motion is simply represented by

$$
\left\{\begin{array}{l}
x^{\prime \prime}+\left(\frac{Q_{x, 0}}{R}\right)^{2} x=0 \\
y^{\prime \prime}+\left(\frac{Q_{y, 0}}{R}\right)^{2} y=0
\end{array},\right.
$$

where $R$ is the average radius of the circular accelerator. The artificial skew quadrupoles with zero length are distributed along the ring, the $4 \times 4$ transfer matrix between adjacent skew quadrupoles are uncoupled.

Here we take one of Rhic rings as a testbed. Each Rhic ring has three families of correction skew quadrupoles. Their contributions to the coupling coefficient are $120^{\circ}$ apart under appropriate power supply polarizations. So here we artificially insert three zero-length skew quadrupoles equidistantly in the simulation model. The ring's circumference is assumed to be $3833.84 \mathrm{~m}$, the two uncoupled tunes are $\left(Q_{x, 0}, Q_{y, 0}\right)=$ $(28.22,29.23)$, both are the same as Rhic's design.

For each tracking job, one particle with initial horizontal and vertical coordinates is launched at the first skew quadrupole. The successive particle's turn-by-turn $x$ and $y$ data are logged at each skew quadrupole location. Knowing the turn-by-turn $(x, y)$ data at one observation point, the 6 quantities defined above can be obtained. In the following we will adopt fast Fourier transformation (FFT) technique to get them. The eigentunes are obtained from FFT of 8192 turns' $(x+y)$ data. The 4 phases $\phi_{i, j}$ are obtained from FFT imaginary and real part's at the eigentunes obtained from FFT of $(x+y)$ turn-by-turn data. The amplitude ratios are from the eigentune peak heights in the FFT plot.

\section{$1.3 \quad$ 1-D global decoupling scan}

Here we observe the 6 quantities' changes during we scan one skew quadrupole's strength. Before scan, all the skew quadrupole strengths are zero and the lattice is uncoupled. The first skew quarupole is chosen for the scan. The turn-by-turn $(x, y)$ data collected at the third skew quadrupole are used for the data processing.

Fig. 1 gives the two eigentune changes during the scan. We see when the machine approaches to be uncoupled, the tune split $\left|Q_{2}-Q_{1}\right|$ keeps decreasing. However, the tune split decreases very small when close to the tune split minimum, or the globally uncoupled point. It is found that in the experiment it is difficult to determine the uncoupled point with a not high resolution tune measurement instrumentation.

Fig. 2 shows the two amplitude ratios $r_{1,2}$ during the scan. We see that the ratios $r_{1,2}$ are very close during the scan. And both of them drop to zero drastically when the scan goes to the uncoupled point. Comparing to the tune split, the ratios are more sensitive for the decoupling detecting when close to the uncoupled point. When the machine is well decoupled, $r_{1,2}$ are close to zero.

Fig. 3 and Fig. 4 show the two phase differences $\Delta \phi_{1}$ and $\Delta \phi_{2}$ during the scan. It is very attractive that they both keep constant or almost constant during the scan, which is possibly useful for the constructing of a phase loop for the global decoupling. There is a phase difference sharp jump $\pi$ across the uncoupled point where the scanning skew quadruole strength equals zero, or in other word, when the scan crosses the uncoupled point. Under the uncoupled situation, the phase differences $\Delta \phi_{1}$ and $\Delta \phi_{2}$ have no meanings. The not-zero values at the uncoupled point in Fig. 3 and Fig. 4 comes from the data processing.

\section{$1.4 \quad 2-\mathrm{D}$ global decoupling scan}

Here we turn to 2-D decoupling scan. Before the scan, the accelerator model is set to be coupled, where the first skew quadrupole's integrated strength is $\left(k_{s} d l\right)_{1}=0.005 \mathrm{~m}^{-1}$. We scan the second and the third skew quadrupole strengths to observe the changes of these 6 quantities during the scan. According the skew 
quadrupole symmetric locations, the strengths of the second and the third skew quadrupoles both should be $\left(k_{s} d l\right)_{2,3}=0.005 \mathrm{~m}^{-1}$ at the globally uncoupled point.

Fig. 5 shows the tune split $\left|Q_{1}-Q_{2}\right|$. Similar to the above 1-D scan, the tune split $\left|Q_{1}-Q_{2}\right|$ decrease to its minimum very slowly when approaching to the uncoupled point. Fig. 6 shows amplitude ratios $r_{1,2}$ 's changes during the scan. Also similar to the above 1-D scan, they decrease to zero more drastically while the scan approaches to the uncoupled point. Fig. 5 and Fig. 6 both show the uncoupled point is located at $\left(k_{s} d l\right)_{2,3}=0.005 \mathrm{~m}^{-1}$.

Fig. 7 and Fig. 8 show the phase difference $\Delta \phi_{1,2}$ during the 2-D scan. We notice that the phase differences keep constant during the scan if the scan is in the right direction to the uncoupled point! And when one direction scan is crossing the uncoupled point, there is a phase jump $\pi$.

Or in other word, in order to decouple the coupled machine, we first measure the phase differences $\Delta \phi_{1,2}$. Then do the skew quadrupole scan to search the uncoupled point while keeping the phase differences unchanged. When a $\pi$ phase jump is encountered, or the drastic phase jump is encountered in that scan direction, the uncoupled point is found. This feature has a very significance for the global decoupling loop or feedback.

And also it is noticed that the right direction to the uncoupled point from one original coupled point doesn't change during the scan to the uncoupled point. So the right direction is the shortest way to the uncoupled point. The right direction can be determined from the experiment by performing a 'circular scan' around the original coupled point, or from the accelerator model based on the following analysis.

If the decoupling searching direction is not the right direction to the uncoupled point, the phase differences will keep on decreasing or increasing. Fig. 9 and Fig. 10 show the phase differences $\Delta \phi_{1,2}$ while scan the first skew quadrupole strength. The accelerator model is originally coupled by setting the second skew quadrupole strength to be $\left(k_{s} d l\right)_{2}=0.005 \mathrm{~m}^{-1}$. As we know, it is impossible to decouple the accelerator model only with the first skew quadrupole strength.

\section{Analysis}

In this section we will give the analytical solutions to the amplitude ratios $r_{1,2}$ and the phase differences $\Delta \phi_{1,2}$ for the free oscillation. There are several linear coupling's parameterizations. Hamiltonian perturbation theory is an approximate treatment, but it supplies more physical insights.

\subsection{Action-angle parameterization approach}

The linear coupling's action-angle parameterization [7] is useful for the analytical calculations and the turnby-turn BPM data interpretations. In the parameterization, the particle's 4 coordinates in the laboratory frame are described as

$$
\begin{gathered}
\left(\begin{array}{c}
x \\
x^{\prime} \\
y \\
y^{\prime}
\end{array}\right)=\mathbf{P} \cdot\left(\begin{array}{c}
\sqrt{2 J_{1}} \cos \Phi_{1} \\
-\sqrt{2 J_{1}} \sin \Phi_{1} \\
\sqrt{2 J_{2}} \cos \Phi_{2} \\
-\sqrt{2 J_{2}} \sin \Phi_{2}
\end{array}\right), \\
\mathbf{P}=\left(\begin{array}{cccc}
p_{11} & 0 & p_{13} & p_{14} \\
p_{21} & p_{22} & p_{23} & p_{24} \\
p_{31} & p_{32} & p_{33} & 0 \\
p_{41} & p_{42} & p_{43} & p_{44}
\end{array}\right),
\end{gathered}
$$

where $J_{1,2}, \Phi_{1,2}$ are the actions and betatron phases of the two eigenmodes, silimar to that defined in the uncoupled one-dimensional situation [8].

It is proved that the matrix $\mathbf{P}$ can be derived from the eigenvectors of the one-turn transfer map. And it also has tight connections to Twiss and coupling paramters defined in Edwards-Teng's linear coupling parameterization $[9,10,11]$,

$$
\mathbf{P}=\left(\begin{array}{cccc}
r \sqrt{\beta_{1}} & 0 & c_{11} \sqrt{\beta_{2}}-c_{12} \alpha_{2} / \sqrt{\beta_{2}} & -c_{12} / \sqrt{\beta_{2}} \\
-\alpha_{1} r / \sqrt{\beta_{1}} & r / \sqrt{\beta_{1}} & c_{21} \sqrt{\beta_{2}}-c_{22} \alpha_{2} / \sqrt{\beta_{2}} & c_{22} / \sqrt{\beta_{2}} \\
-c_{12} \alpha_{1} / \sqrt{\beta_{1}}-c_{22} \sqrt{\beta_{1}} & c_{12} / \sqrt{\beta_{1}} & r \sqrt{\beta_{2}} & 0 \\
c_{11} \alpha_{1} / \sqrt{\beta_{1}}+c_{21} \sqrt{\beta_{1}} & -c_{11} / \sqrt{\beta_{1}} & -\alpha_{2} r / \sqrt{\beta_{2}} & r / \sqrt{\beta_{2}}
\end{array}\right) .
$$

In order to easily distinguish Twiss parameter $\gamma$, here we use $r$ to take place of the coupling parameter $\gamma$ in other literatures. 
$x$ and $y$ coordinates are described as

$$
\left\{\begin{array}{l}
x=p_{11} \sqrt{2 J_{1}} \cos \Phi_{1}+p_{13} \sqrt{2 J_{2}} \cos \Phi_{2}-p_{14} \sqrt{2 J_{2}} \sin \Phi_{2} \\
y=p_{31} \sqrt{2 J_{1}} \cos \Phi_{1}-p_{32} \sqrt{2 J_{1}} \sin \Phi_{1}+p_{33} \sqrt{2 J_{2}} \cos \Phi_{2}
\end{array} .\right.
$$

Then the above defined $r_{1,2}$ and $\Delta \phi_{1,2}$ are obtained

$$
\begin{gathered}
\left\{\begin{array}{c}
r_{1}=\sqrt{p_{31}^{2}+p_{32}^{2}} / p_{11} \\
r_{2}=\sqrt{p_{13}^{2}+p_{14}^{2}} / p_{33}
\end{array}\right. \\
\left\{\begin{array}{c}
\Delta \phi_{1,0}=\arctan \left(p_{32} / p_{31}\right) \\
\Delta \phi_{2,0}=\arctan \left(p_{14} / p_{13}\right)
\end{array} .\right.
\end{gathered}
$$

or in the Twiss and coupling parameters,

$$
\begin{gathered}
\left\{\begin{array}{l}
r_{1}=\sqrt{\beta_{1} c_{22}^{2}+2 \alpha_{1} c_{22} c_{12}+\gamma_{1} c_{12}} /\left(r \sqrt{\beta_{1}}\right) \\
r_{2}=\sqrt{\beta_{2} c_{11}^{2}-2 \alpha_{2} c_{11} c_{12}+\gamma_{2} c_{12}} /\left(r \sqrt{\beta_{2}}\right)
\end{array}\right. \\
\left\{\begin{array}{l}
\Delta \phi_{1,0}=\arctan \left(-c_{12} /\left(\alpha_{1} c_{12}+\beta_{1} c_{22}\right)\right) \\
\Delta \phi_{2,0}=\arctan \left(c_{12} /\left(-\alpha_{2} c_{12}+\beta_{2} c_{11}\right)\right)
\end{array}\right.
\end{gathered}
$$

where we define $\gamma_{1}=\left(1+\alpha_{1}^{2}\right) / \beta_{1}, \gamma_{2}=\left(1+\alpha_{2}^{2}\right) / \beta_{2}$.

These expressions are straight-forward to give $r_{1,2}$ and $\Delta \phi_{1,2}$. However, it requires the knowledges of the optics. Individual skew quadrupole's contributions to these quantities are not reflected. In the following, we will turn to linear coupling's Hamiltonian perturbation theory, where the individual skew quadrupole's contributions to these quantities are transparent.

\subsection{Hamiltonian perturbation theory approach}

Here we follow G. Guignard and Wiedemann's work [12, 13, 14] and figure out the general expression of $x$ and $y$ coordinates. From the expression of $x$ and $y$ coordinates, we obtain the general descriptions of $r_{1,2}$ and $\Delta \phi_{1,2}$.

The equations for the coupled betatron motion is given by

$$
\left\{\begin{array}{l}
x^{\prime \prime}+k_{x}(s) x=-k_{s}(s) y \\
y^{\prime \prime}+k_{y}(s) y=-k_{s}(s) x
\end{array}\right.
$$

where $k_{s}(s)$ is the skew quadrupole strength. Eq. (13) can be derived from the Hamiltonian

$$
H=\frac{1}{2} x^{\prime 2}+\frac{1}{2} k_{x} x^{2}+\frac{1}{2} y^{\prime 2}+\frac{1}{2} k_{y} y^{2}+k_{s}(s) x y,
$$

where $x, x^{\prime}, y, y^{\prime}$ are the four canonical variables. To solve Eq. (13), we apply the method of variation of integration constants and try the ansatz,

$$
\left\{\begin{array}{l}
z(s)=\sqrt{2 a_{z}(s)} \sqrt{\beta_{z}} \cos \left[\Psi_{z}(s)+\phi_{z, 0}(s)\right] \\
z^{\prime}(s)=-\frac{\sqrt{2 a_{z}(s)}}{\sqrt{\beta_{z}(s)}}\left\{\alpha_{z}(s) \cos \left[\Psi_{z}(s)+\phi_{z, 0}(s)\right]+\sin \left[\Psi_{z}(s)+\phi_{z, 0}(s)\right]\right\}
\end{array}\right.
$$

where $a_{z}(s), \phi_{z, 0}, z=x, y$, are the new four canonical variables. $\varphi=2 \pi \cdot s / L$ is the independent variable, $L$ is circumference of the circular accelerator. The new Hamiltonian is

$$
H_{1}=2 k_{s} \sqrt{\beta_{x} \beta_{y}} \sqrt{a_{x} a_{y}} \cos \left(\Psi_{x}+\phi_{x, 0}\right) \cos \left(\Psi_{y}+\phi_{y, 0}\right) .
$$

In the following we will drop $s$ in the following.

Since we assume the weak difference coupling situation, by isolating the linear difference resonance coupling item, we obtain the new Hamiltonian from Eq. (16)

$$
H_{2}=\left|C^{-}\right| \sqrt{a_{x} a_{y}} \cos \left(\Psi_{x}-\Psi_{y}+\Delta \cdot \varphi+\chi\right)
$$


where $\Delta$ is the uncoupled tune decimal part split, or the tune split in the uncoupled situation,

$$
\Delta=Q_{x, 0}-Q_{y, 0}-p,
$$

$p$ is an integer. The coupling coefficient $C^{-}$is defined as

$$
C^{-}=\left|C^{-}\right| e^{i \chi}=\frac{1}{2 \pi} \int_{0}^{L} \sqrt{\beta_{x} \beta_{y}} k_{s} e^{i\left[\Phi_{x}-\Phi_{y}-2 \pi \Delta \cdot s / L\right]} d l .
$$

Please notice that in Ref. [14] the coupling coefficient's phase is not included in $H_{2}$. To eliminate the independent variable $\varphi$ from the Hamiltonian $H_{2}$, we use the generating function

$$
G=\tilde{a}_{x}\left(\phi_{x, 0}+\frac{1}{2} \Delta \cdot \varphi+\frac{1}{2} \chi\right)+\tilde{a}_{y}\left(\phi_{y, 0}-\frac{1}{2} \Delta \cdot \varphi-\frac{1}{2} \chi\right),
$$

we get

$$
\left\{\begin{array}{l}
\tilde{\phi}_{x, 0}=\phi_{x, 0}+\frac{1}{2} \Delta \cdot \varphi+\frac{1}{2} \chi \\
\tilde{\phi}_{y, 0}=\phi_{y, 0}-\frac{1}{2} \Delta \cdot \varphi-\frac{1}{2} \chi
\end{array},\right.
$$

and $\tilde{a}_{x}=a_{x}, \tilde{a}_{y}=a_{y}$. The new Hamiltonian is

$$
H_{3}=\frac{1}{2} \Delta \cdot\left(a_{x}-a_{y}\right)+\left|C^{-}\right| \sqrt{a_{x} a_{y}} \cos \left(\tilde{\phi}_{x, 0}-\tilde{\phi}_{y, 0}\right) .
$$

Following the suggestion by G. Guignard, we define two new variables $w$ and $v, w=\sqrt{a_{x}} e^{i \tilde{\phi}_{x, 0}}$ and $v=\sqrt{a_{y}} e^{i \tilde{\phi}_{y, 0}}$. Then after some manipulations, we get

$$
\left\{\begin{array}{l}
d w / d \varphi=\frac{i}{2}\left(\Delta w+\left|C^{-}\right| v\right) \\
d v / d \varphi=\frac{i}{2}\left(\left|C^{-}\right| w-\Delta v\right)
\end{array} .\right.
$$

Try ansatz

$$
\left\{\begin{array}{l}
w(\varphi)=a e^{i \nu \varphi}+b e^{-i \nu \varphi} \\
v(\varphi)=c e^{i \nu \varphi}+d e^{-i \nu \varphi}
\end{array}\right.
$$

we obtain

$$
\nu=\frac{1}{2} \sqrt{\Delta^{2}+\left|C^{-}\right|^{2}}
$$

and

$$
\left\{\begin{array}{l}
\frac{c}{a}=\frac{\left|C^{-}\right|}{2 \nu+\Delta} \\
\frac{b}{d}=-\frac{\left|C^{-}\right|}{2 \nu+\Delta}
\end{array} .\right.
$$

Then finally we are able to re-write the particle's $x$ and $y$ coordinates in the two eigenmodes as

$$
\left\{\begin{array}{l}
\left.\left.x(s)=\sqrt{2 \beta_{x}}\left\{a \cos \left[\Psi_{x}+(\nu-\Delta / 2) \varphi-\chi / 2\right)\right]+b \cos \left[\Psi_{x}-(\nu+\Delta / 2) \varphi-\chi / 2\right)\right]\right\} \\
\left.\left.y(s)=\sqrt{2 \beta_{y}}\left\{c \cos \left[\Psi_{y}+(\nu+\Delta / 2) \varphi+\chi / 2\right)\right]+d \cos \left[\Psi_{y}-(\nu-\Delta / 2) \varphi+\chi / 2\right)\right]\right\}
\end{array} .\right.
$$

There are two eigentunes in Eq. (27),

$$
\left\{\begin{array}{l}
Q_{1}=Q_{x, 0}-\frac{1}{2} \Delta+\frac{1}{2} \sqrt{\Delta^{2}+\left|C^{-}\right|^{2}} \\
Q_{2}=Q_{y, 0}+\frac{1}{2} \Delta-\frac{1}{2} \sqrt{\Delta^{2}+\mid C^{-\left.\right|^{2}}}
\end{array} .\right.
$$

Therefore the tune split is

$$
\left|Q_{1}-Q_{2}\right|=\sqrt{\Delta^{2}+\left|C^{-}\right|^{2}} .
$$

Comparing Eq. (27) to Eq. (1), we get $\Delta \phi_{1,2}$ and $r_{1,2}$,

$$
\begin{gathered}
\left\{\begin{array}{l}
r_{1}=\sqrt{\frac{\beta_{y}}{\beta_{x}}} \cdot \frac{\left|C^{-}\right|}{2 \nu+\Delta} \\
r_{2}=\sqrt{\frac{\beta_{x}}{\beta_{y}}} \cdot \frac{\left|C^{-}\right|}{2 \nu+\Delta}
\end{array},\right. \\
\left\{\begin{array}{l}
\Delta \phi_{1}=\chi \\
\Delta \phi_{2}= \pm \pi-\chi
\end{array}\right.
\end{gathered}
$$


Now we have obtained the expressions of the 6 quantities $Q_{1,2}, r_{1,2}$ and $\Delta \phi_{1,2}$ defined above. In Wiedemann's work [14], the general expression for $x$ and $y$ are not given. And as mentioned before, the coupling phase is not included in Hamiltonian $\mathrm{H}_{2}$, so the phase differences Eq. (31) was not obtained there.

Comparing to that from the matrix approach, Eq. (30) and Eq. (31) from the Hamiltonian perturbation theory are more convenient for the interpreting $r_{1,2}$ and $\Delta \phi_{1,2}$ since the coupling coefficient is connected to the individual skew quadrupole. The demanding readers are encouraged to interpret the observations in the above 1-D and 2-D skew quadrupole strength scan with Eq. (19), Eq. (30) and Eq. (31).

\section{Possible phase loop for global decoupling}

\subsection{Phase loop proposal}

From Eq. (31), we find that the phase differences $\Delta \phi_{1}$ equals to the phase of the coupling coefficient $C^{-}$. For $\Delta \phi_{2}$, it equal to $( \pm \pi-\chi)$. In order to decouple the coupled machine, we then need to keep the phase of the total coupling coefficient in the machine, or keep the observed phase difference $\Delta \phi_{1}$, and reduce and eventually cancel the residual coupling's amplitude. This implies a loop for the global coupling correction loop based on the phase difference $\Delta \phi_{1}$.

As we mentioned before, In the experiment we first measure the phase differences $\Delta \phi_{1,2}$. Then do the skew quadrupole scan to search the uncoupled point while keeping the phase differences unchanged. When a $\pi$ phase jump is encountered, or the drastic phase jump is encountered in the scan direction, the uncoupled point is found. The right direction can be determined from the experiment by performing 'circular scan' around the original coupled point, or from the accelerator model based on Eq. (19).

Generally there are two orthognal skew quadrupole correction families for the global decoupling. If the accelerator's optics is not severely biased from the model, it is easy to get the two family skew quadrupole's strength ratio to obtain the wanted coupling coefficient phase $\chi$.

Other observables like $\left|Q_{1}-Q_{2}\right|, r_{1,2}$ are also helpful during the experimental searching for the right decoupling direction. They can be used to verify or cross-check the right decoupling direction in the phase decoupling loop.

It should be mentioned that the coupling coefficient is location dependent quantity. It is easy to prove that the amplitude of coupling coefficient is a global constant. From one observation point to another, the phase of the coupling coefficient will change. The phase change is $\left(\Delta \Phi_{x}-\Delta \Phi_{y}-\frac{2 \pi d s}{L} \cdot \Delta\right)$, where $\Delta \Phi_{x, y}$ are the betatron phase advances between the two points, $d s$ is the distance between them. $\Delta$ is the decimal uncoupled tune split defined above. So it is not necessary to observe the phase differences $\Delta \phi_{1,2}$ along the ring.

\subsection{PLL driving oscillation}

Since above analytical solutions and simulations are all based on the free oscillation. To paractically make use of the phase loop for the global decoupling, we still need to extend the above simulation and analytical solutions to the driving oscillation. The key point is what the difference of the phase differences observed from the free oscillation and the PLL driving oscillation. This work has been started. And the dedicated beam experiment is proposed for the coming Rhic'05 run. The results will be reported in the following notes.

\section{Acknowledgments}

The authors thank T. Roser for discussion of the differences between the free oscillation and the driving oscillation. And thank R. Talman (Cornell) for the discussion of local and global observables of linear coupling. One of the authors Y. Luo also wants to thank C. Gardner for the discussion of the matrix approximation approaches and the experimental BPM turn-by-turn data interpretations in the weak coupling situation. This work is support by the US DOE under contract No. DE-AC02-98CH10886.

\section{References}

[1] P.castro, et al., PAC 1993.

[2] J. Irwin, et al., Phys. Rev. Lett. 82, p.1684 (1999). 
[3] C.-x. Wang, et al., Phys. Rev. ST Accel. Beams 6, 104001(2003)

[4] D. Sagan, Phys. Rev. ST Accel. Beam 4, 122801 (2001).

[5] D. Sagan, R. Meller, et al., Phys. Rev. ST Accel. Beam 3, 102801 (2000).

[6] A. Chao, Collective Effects in High Energy Accelerators, John Wiley \& Sons, INC., 1993.

[7] Y. Luo, Linear coupling's parameterization in the rotation frame, RHIC-AP-165.

[8] E.D. Courant, H.S. Snyder, Ann. Phys. 3 (1958).

[9] D. Edwards, L. Teng, PAC 1973, p.885.

[10] M. Billing, Cornell Report CBN 85-2 ( 1985 ).

[11] D. Sagan, D. Rubin, Phys. Rev. ST Accel. Beam 2, 074001 (1999).

[12] G. Guignard, CERN Report No. 76-06, 1976.

[13] G. Guignard, Phys. Rev. E 51, p6104, 1995.

[14] H. Wiedemann, Particle Accelerator Physics II, Nonlinear and Higher-Order Beam Dynamics, SpringerVerlag, 1995. 


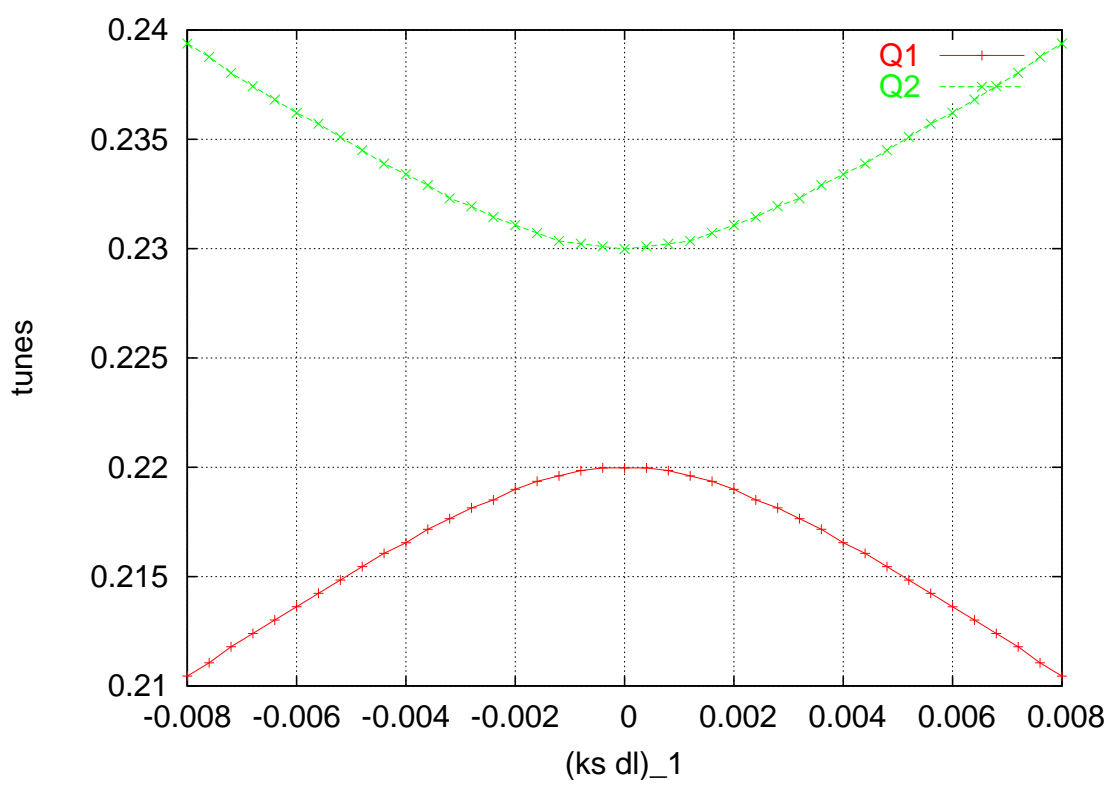

Figure 1: The eigentunes $Q_{1,2}$ in the 1-D decoupling scan.

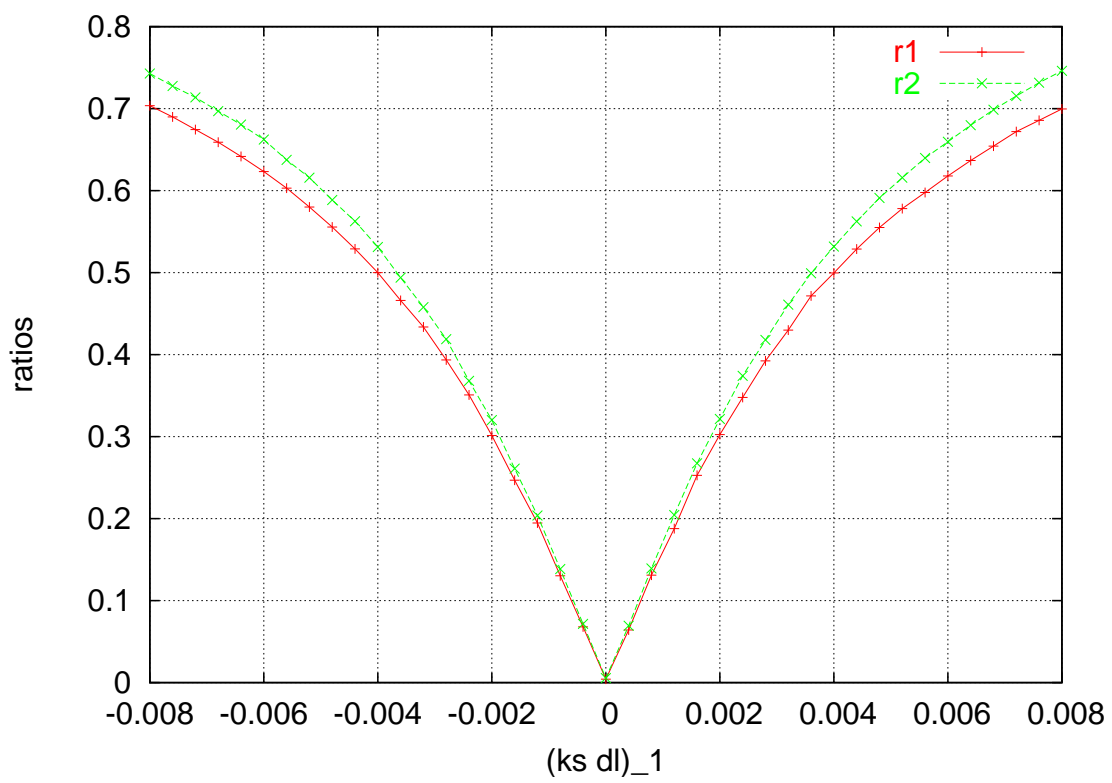

Figure 2: The amplitude ratios $r_{1,2}$ in the 1-D decoupling scan. 


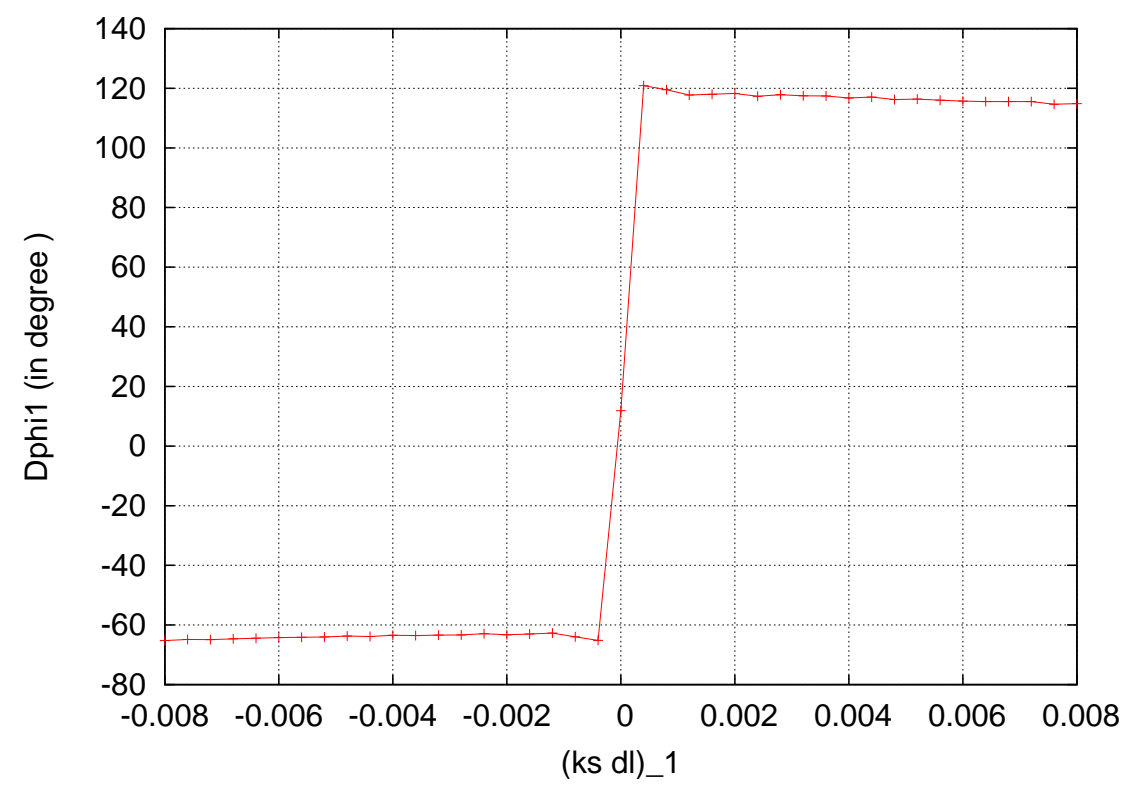

Figure 3: The phase differences $\Delta \phi_{1}$ in the 1-D decoupling scan.

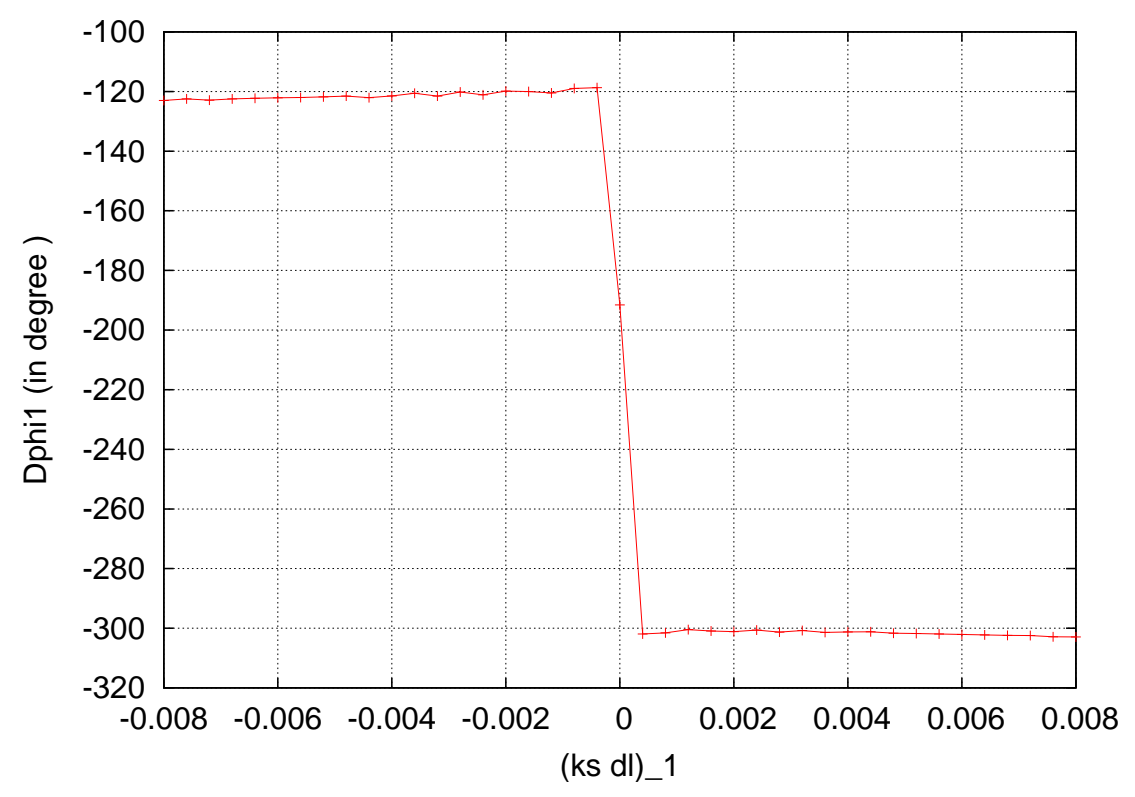

Figure 4: The phase differences $\Delta \phi_{2}$ in the 1-D decoupling scan. 


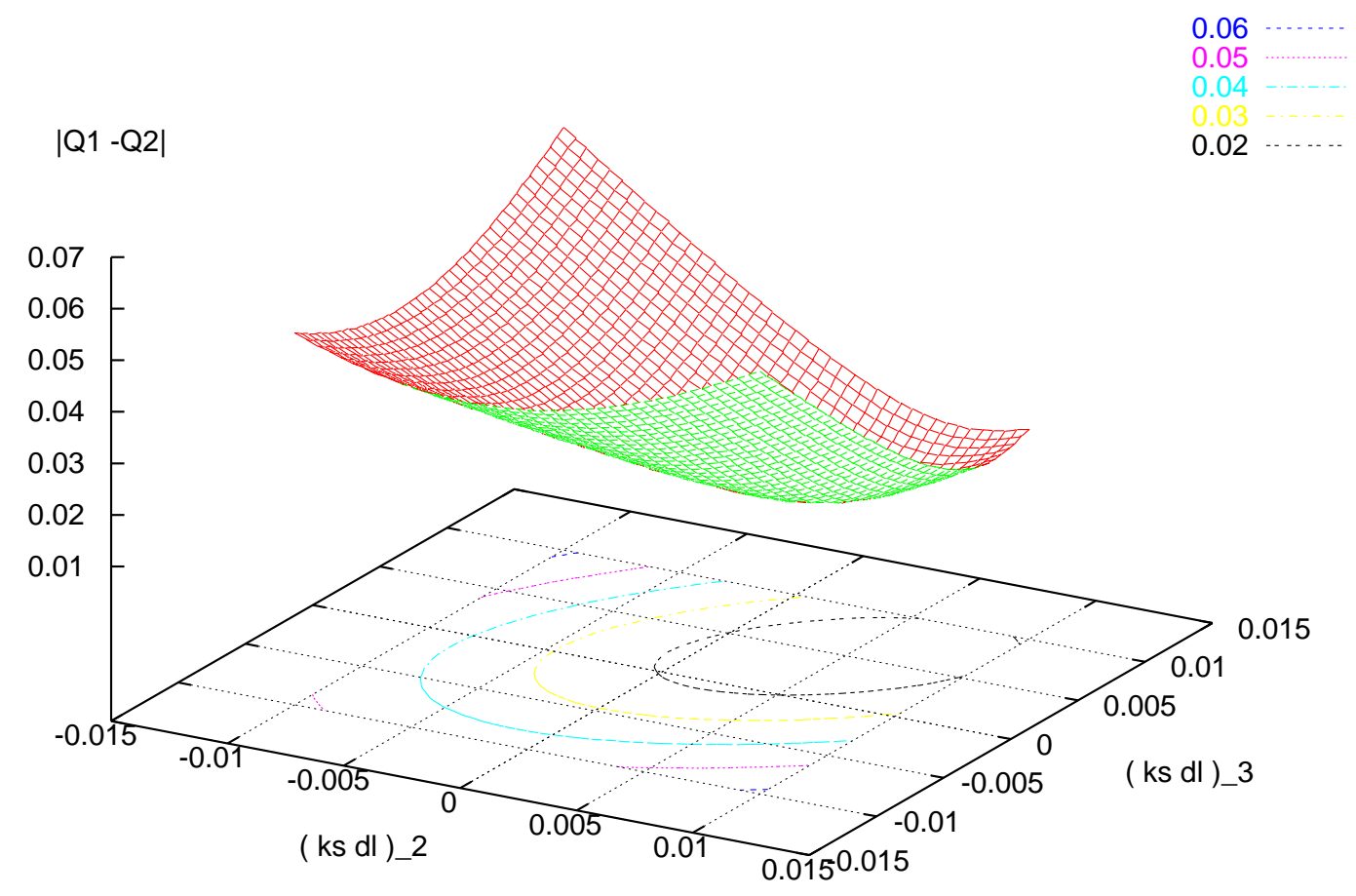

Figure 5: The tune split $\left|Q_{1}-Q_{2}\right|$ in the 2-D decoupling scan.

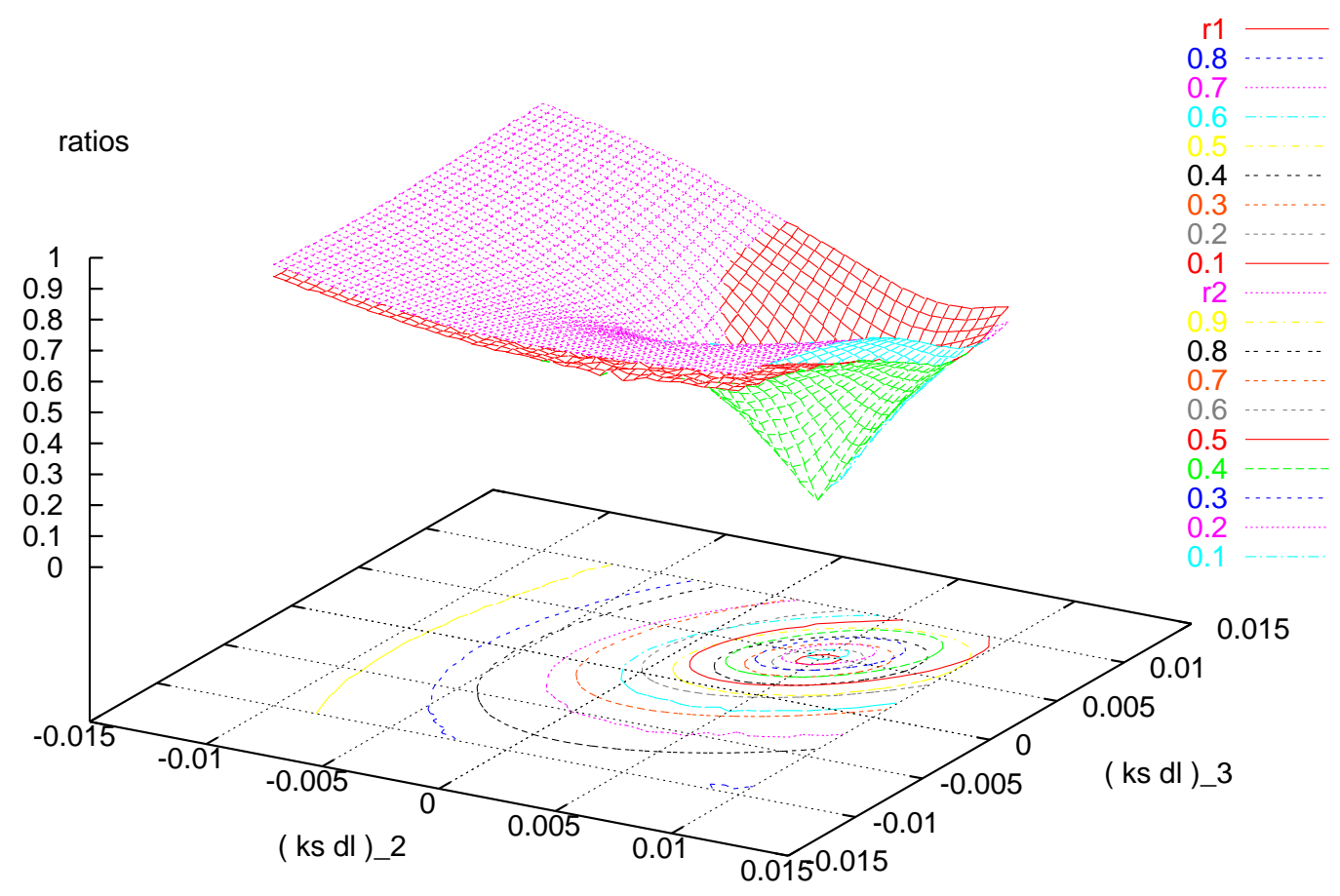

Figure 6: The amplitude ratios $r_{1,2}$ in the 2-D decoupling scan. 


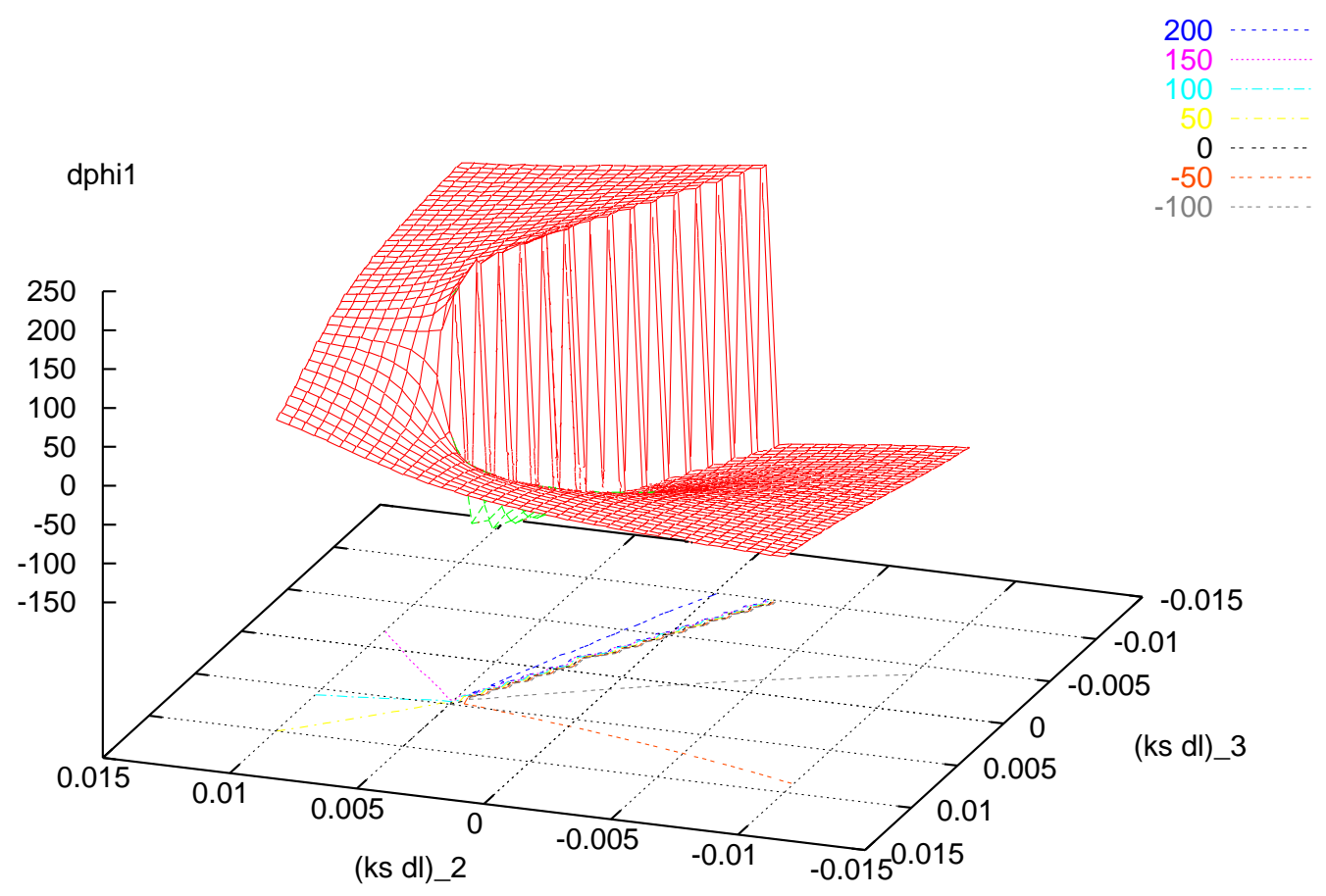

Figure 7: The phase differences $\Delta \phi_{1}$ in the 2-D decoupling scan.

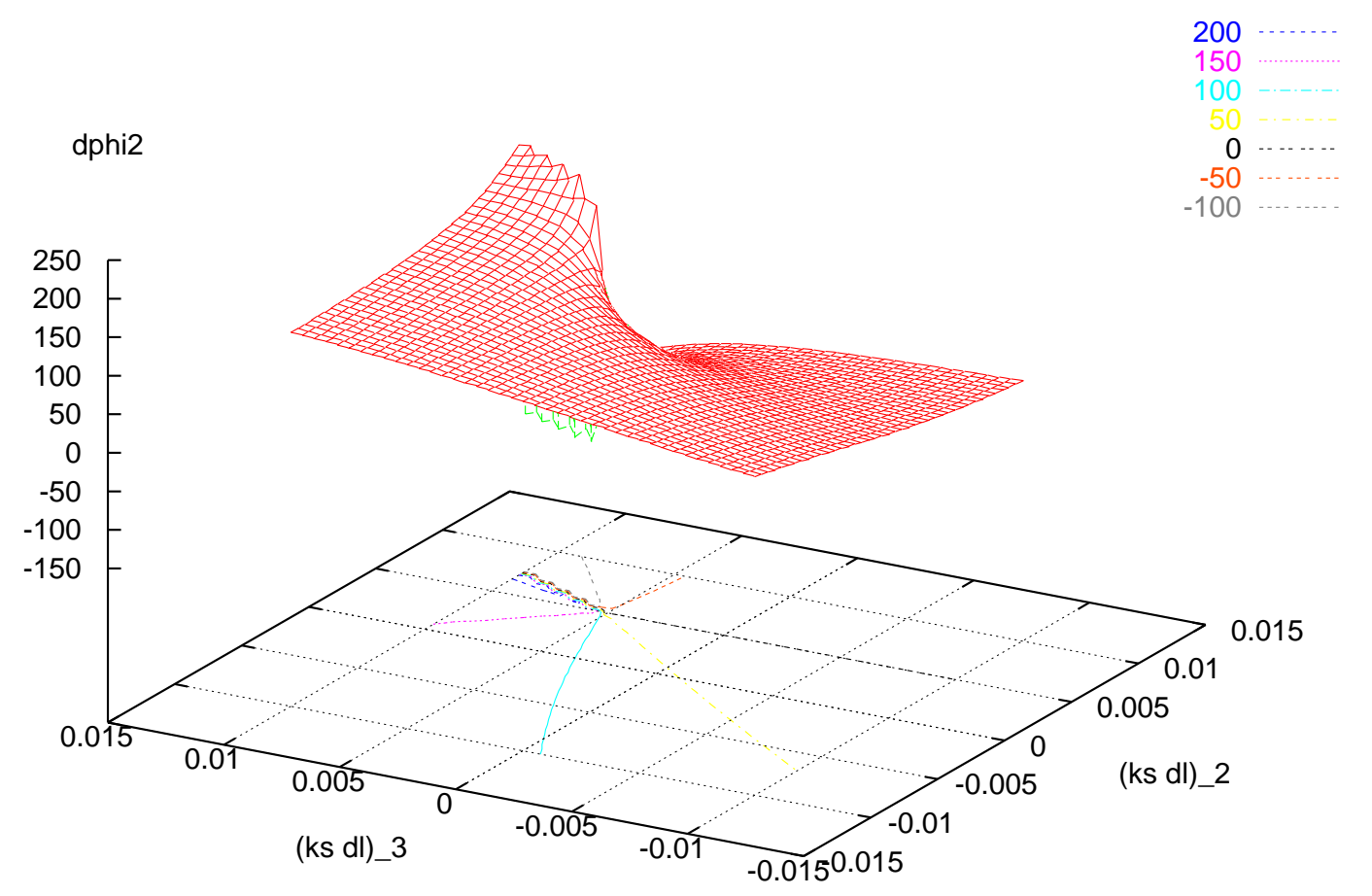

Figure 8: The phase differences $\Delta \phi_{2}$ in the 2-D decoupling scan. 


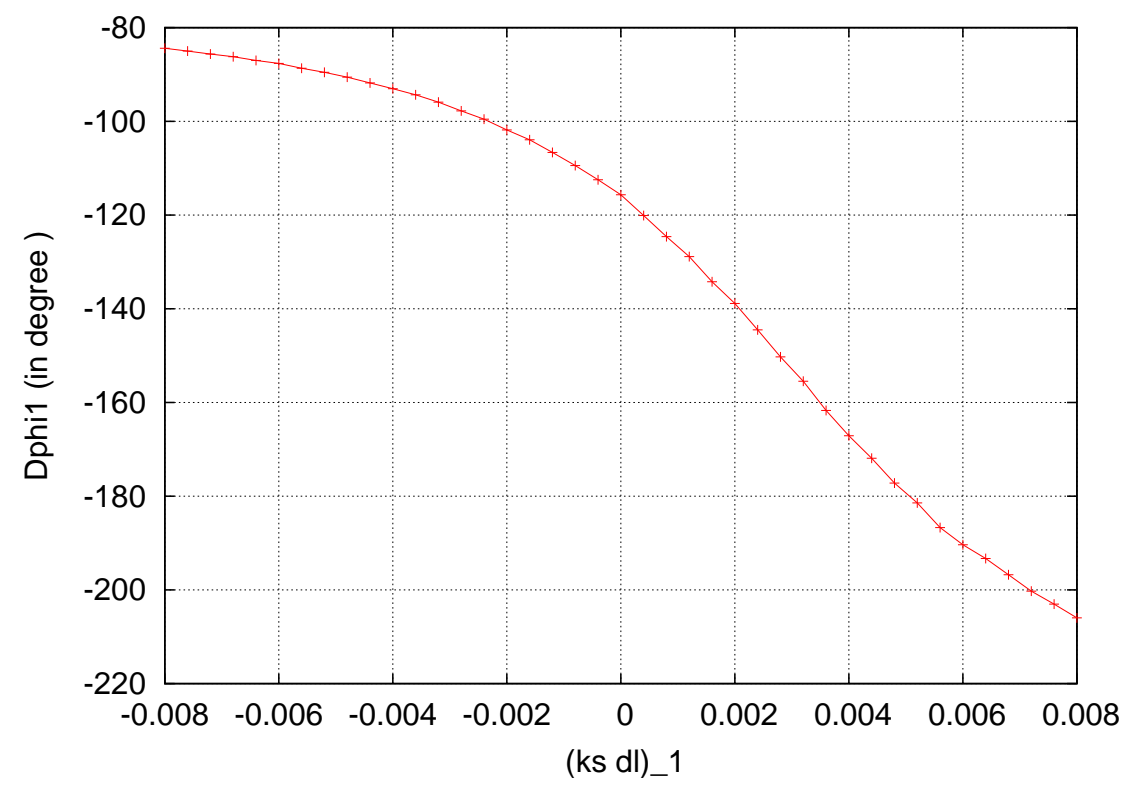

Figure 9: The phase differences $\Delta \phi_{1}$ in the wrong scan direction.

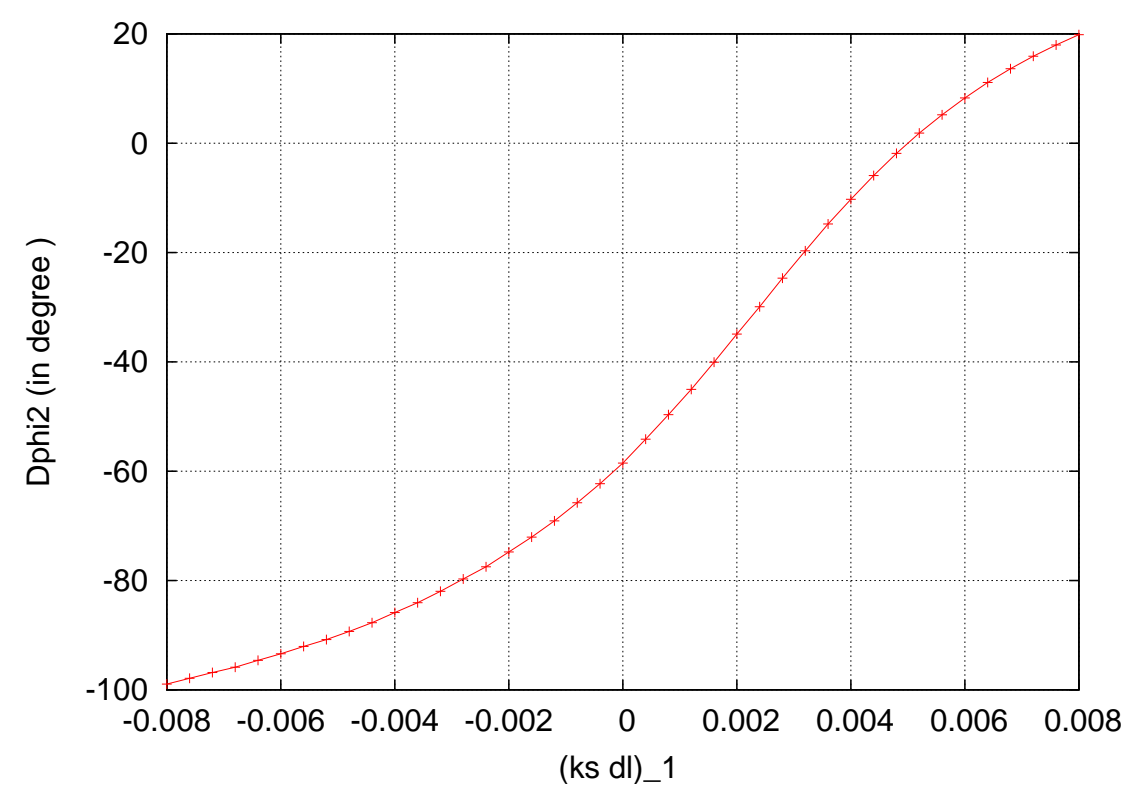

Figure 10: The phase differences $\Delta \phi_{2}$ in the wrong scan direction. 